\title{
Passive and catalytic antibodies and drug delivery*
}

\author{
G. Michael Blackburn ${ }^{1, \ddagger}$, J. H. Rickard ${ }^{1}$, S. Cesaro-Tadic ${ }^{2}$, D. Lagos ${ }^{3}$, \\ A. Mekhalfia ${ }^{1}$, L. Partridge ${ }^{3}$, and A. Plückthun ${ }^{2}$ \\ ${ }^{1}$ Krebs Institute, Chemistry Department, Sheffield University, Sheffield, S3 7HF, \\ UK; ${ }^{2}$ Biochemisches Institut, Universität Zürich, Winterthurerstrasse 190, CH-8057 \\ Zurich, Switzerland; ${ }^{3}$ Krebs Institute, Department of Molecular Biology and \\ Biotechnology, University of Sheffield, Sheffield, S10 2TN, UK
}

\begin{abstract}
Antibodies are one of the most promising components of the biotechnology repertoire for the purpose of drug delivery. On the one hand, they are proven agents for cell-selective delivery of highly toxic agents in a small but expanding number of cases. This technology calls for the covalent attachment of the cytotoxin to a tumor-specific antibody by a linkage that is reversible under appropriate conditions (antibody conjugate therapy, ACT"passive delivery"). On the other hand, the linker cleavage can be accomplished by a protein catalyst attached to the tumor-specific antibody ("catalytic delivery"). Where the catalyst is an enzyme, this approach is known as antibody-directed enzyme prodrug therapy (ADEPT). Where the transformation is brought about by a catalytic antibody, it has been termed antibody-directed abzyme prodrug therapy (ADAPT). These approaches will be illustrated with emphasis on how their demand for new biotechnology is being realized by structure-based protein engineering.
\end{abstract}

\section{INTRODUCTION}

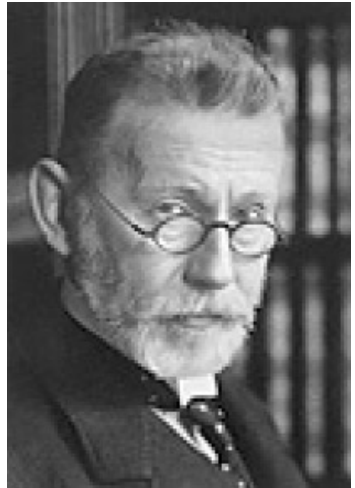

Paul Ehrlich

The origin of the "magic bullet" concept of selective delivery of a cytotoxic agent to tumor cells originates from the imagination of Paul Ehrlich (left inset) (Nobel Laureate, 1908). His concept arose from the experience of $19^{\text {th }}$ century German chemists who selectively stained tissues for histological examination. Ehrlich argued that if a compound could be made that selectively targeted a disease-causing organism, then a toxin for that organism could be delivered along with the agent of selectivity. Hence, a "magic bullet" would be created that killed only the organism targeted [1]. For many years, the sought-for means of delivery was thought to lie in cell-selective dyestuffs. However, a completely new approach was created by the discovery of monoclonal antibodies by Cesar Milstein and Georges Köhler (Nobel Prize, 1984) [2]. In particular, Milstein demonstrated that spleen cells from mice immunized with rat thymocyte membranes and fused with a mouse myeloma led to hybridomas with the specific ability to detect different, previously undefined antigens on different subpopulations of lymphoid cells. Some of these hybrids were selected and screened for the production of antibodies to rat thymocytes by a binding assay.

\footnotetext{
*Lecture presented at the Polish-Austrian-German-Hungarian-Italian Joint Meeting on Medicinal Chemistry, Kraków, Poland, 15-18 October 2003. Other presentations are published in this issue, pp. 907-1032.

${ }^{\ddagger}$ Corresponding author
} 


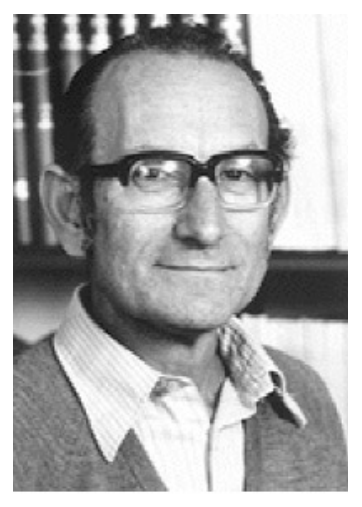

Cesar Milstein

Between 5000 and 36000 antibody molecules per cell could be bound at saturation to different cell markers on the thymocytes. The method is therefore extremely sensitive and permits selective identification down to minor membrane molecules and also of antigens on small subpopulations of a heterogeneous mixture of cells [3].

In the almost three decades since the development of these methods for preparing monoclonal antibodies, we are finally beginning to see their fruits for cancer therapy [4]. Early ideas were that "naked" tumor-specific antibodies might lead to cell clearance directly. Later work sought to use tumorspecific antibodies to deliver radioactive isotopes (e.g., $\mathrm{I}^{131}$ ) to cause selective tumor cell killing by localized high-energy radioactive emission. More success has attended work on two aspects of antibody delivery of cytotoxic agents.

In antibody-directed therapy (ADT), the toxin is covalently bonded to the antibody to be released after reaching the target tumor cell. From a large number of toxins explored, one product has gained FDA approval. Work originating in Seattle focused on the treatment of acute myeloid leukemia (AML) with antibodies specific for a CD33 cell surface marker that is characteristic in some $20 \%$ of AML cases. AML is the most common type of acute leukemia in adults. Although conventional chemotherapy induces clinical remissions in most patients with AML, recurrent leukemia represents the major obstacle to cure. Chemotherapy specifically targeted to leukemic cells by monoclonal antibodies might enable patients to achieve remissions more safely than using conventional approaches. After evaluating a series of phase II studies, the U.S. Food and Drug Administration approved Mylotarg ${ }^{\mathrm{TM}}$ (gemtuzumab ozogamicin) for the treatment of patients with CD33-positive AML in first relapse who are 60 years or older and who are not considered candidates for other types of cytotoxic chemotherapy. Mylotarg has been jointly developed and launched by Celltech Group and the Wyeth-Ayerst Research division of American Home Products as a treatment for AML. Possible first-line therapy could follow depending on further studies, creating a potential market of USD 300 million.

Gemtuzumab ozogamicin, GO, is a bioconjugate of a humanized anti-CD33 antibody with the ene-diyne calicheamicin $\gamma_{1}$. Calicheamicin (Fig. 1) is a minor DNA groove-binder that causes doublestranded DNA breaks and apoptosis of the target cells, and is too dangerously toxic for direct clinical use. However, the GO bioconjugate selectively targets CD33 markers, internalizes into the AML cells, and releases calicheamicin to penetrate the nucleus and there cause unrepairable DNA damage leading to apoptosis (Fig. 1).

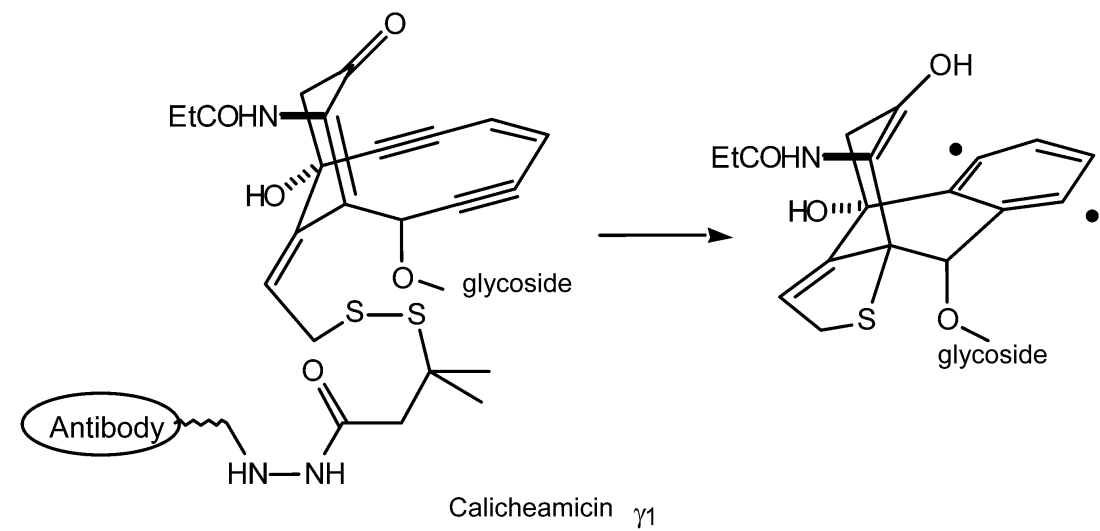

Fig. 1 Calicheamicin is activated by cleavage of the $\mathrm{S}-\mathrm{S}$ bond. The released thiol adds to the $\alpha, \beta$-unsaturated ketone bringing about a conformational change which enables the Bergman rearrangement. This generated the benzene1,4-diradical that abstracts two hydrogen atoms from proximate residues in DNA causing a double-strand break. 
The second use of antibody delivery involves the catalyzed production of the toxic agent at the tumor site by transformation of an inactive prodrug. Such antibody-directed enzyme prodrug therapy (ADEPT) was conceived by Ken Bagshawe [6] and has been developed in many laboratories, particularly in Astra-Zeneca UK. His ADEPT approach consists of three stages: administration of an antibodyenzyme conjugate; subsequent administration of a clearing antibody to eliminate unbound antibody conjugate from the periphery; and administration of prodrug 2-3 d after the conjugate. Zeneca has focused on a conjugate of a bacterial carboxypeptidase CPG2 to an antibody raised to a colorectal tumor cell marker. The preferred prodrug was a benzoic acid mustard linked as an amide to L-glutamic acid (Fig. 2). Hydrolysis of the amide by CPG2 released the mustard with an increase in activity of some 15 -fold. This process proved able to kill human LS147T colorectal tumor xenografts in mice using an ADEPT regimen [7] (Fig. 2).

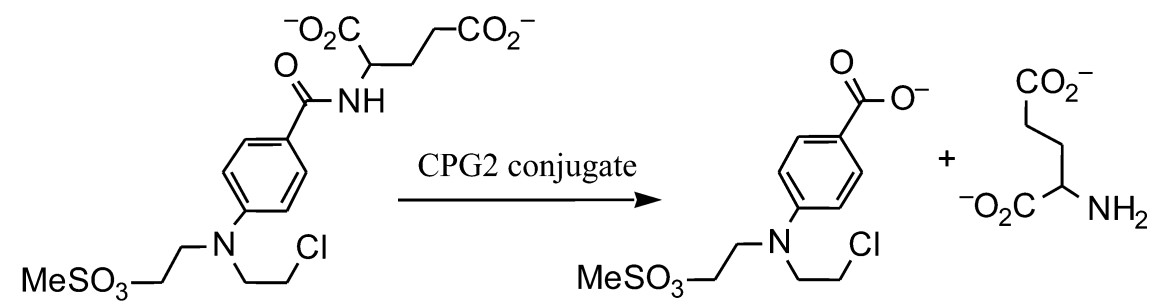

Fig. 2 Conversion of a benzoic acid glutamate amide prodrug into an active benzoic acid mustard by carboxypeptidase CPG2.

\section{RESULTS}

ADEPT has limitations, and these include the response of the patient's immune system to the two proteins: the tumor-specific fragment antibody and the bacterial carboxypeptidase. In one approach to resolving these problems, we sought to generate a human (or humanized) catalytic antibody capable of converting a prodrug into the cytotoxic agent. However, catalytic antibodies have to date proved ineffective at cleaving unactivated amides, such as the Zeneca prodrug 4-[(2-chloroethyl),(2-mesyloxyethyl)-amino]-benzoyl-L-glutamic acid (Fig. 2). We therefore elected to generate a carbamatase antibody that could operate on a carbamate prodrug (Fig. 3). We named the resulting process "ADAPT", being antibody-directed abzyme prodrug therapy [8].<smiles>O=C(Nc1cc([N+](=O)[O-])cc([N+](=O)[O-])c1)Oc1ccc(N(CCCl)CCCl)cc1</smiles><smiles>O=C([O-])c1cc(NC([O-])(O)Oc2ccc(N(CCO)CCCl)cc2)cc(C(=O)[O-])c1</smiles>

(b)

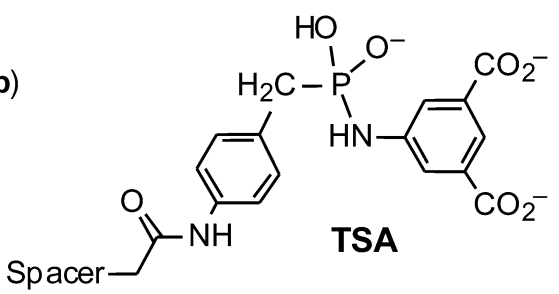<smiles>[O-]c1ccc(N(CC[AlH2])CC[Hg])cc1</smiles>

Fig. 3 (a) Carbamate prodrug and its conversion via a $\mathrm{B}_{\mathrm{Ac}} 2$ mechanism into the phenolic mustard; (b) transitionstate analog. 
We proposed to enhance the disfavored $\mathrm{B}_{\mathrm{Ac}}{ }^{2}$ mechanism of carbamate cleavage, which is normally some two decades slower than the favored E1cb mechanism simply through consideration of the problems of generating a stable transition-state analog (TSA) for the latter process. Paul Wentworth designed and synthesized a phosphonamide TSA for prodrug cleavage (Fig. 3), used it to immunize BalbC mice, and raised a large number of monoclonal antibodies with good binding affinity for the TSA. Several of these antibodies proved capable of effective hydrolysis of the carbamate linkage in 4-nitrophenyloxycarbonylaminoisophthalate, while others showed significant cell-kill activity for treatment of human LoVo cells in tissue culture. Disappointingly, there was no direct correlation between such activity and the results of a cell-kill assay in vitro [8]. Nonetheless, a mechanistic analysis of selected catalytic antibodies established that, as scheduled, they promoted the disfavored $\mathrm{B}_{\mathrm{Ac}} 2$ mechanism by some 6-7 decades relative to the uncatalyzed reaction rate. DF8D5 thus emerged as one of the most effective catalytic antibodies generated in the mid-1990s [10]. However, its performance (as IgG or Fab) was still two slow for in vivo development, and the general $K_{\mathrm{m}}$ of such abzymes for the prodrug was on the high side for potential therapeutic use.

We therefore sought improvements within the manifold of production of catalytic antibodies using murine immunization with TSAs but with very limited success. It became apparent that our task called for new chemistry and a new biotechnology. In particular, the use of the mouse as a source of monoclonal antibodies had proved too uncertain in our hands and in the hands of others, in addition to being a time-consuming and expensive approach to the task.

The whole process of finding catalytic antibodies among the murine immunoglobulin repertoire by screening for turnover has to be one of the most inefficient processes imaginable. It has been brought into limited viability by reducing the size of the protein repertoire from some 10 million candidates to a few hundred by selective induction of their production using stable transition-state analogs a immunogens. That process itself relies on the insights of Linus Pauling [11] and Bill Jencks [12] and is based on the expectation that a protein that has high affinity for a TSA will be a catalyst for the respective reaction. While this remains a strong principle of how enzymes work, it has not proved to be strongly validated by an analysis [13] of the performance of catalytic antibodies using the simple, derived relationship:

$$
\log k_{\text {cat }}-\log k_{\text {uncat }}=\log K_{\mathrm{m}}-\log K_{\mathrm{i}} \text {. }
$$

We therefore reconsidered the basic requirements for identification and production of a protein catalyst and came up with the following specifications:

- $\quad$ access to much larger protein libraries, not necessarily of immunoglobulin proteins;

- $\quad$ employment of selection rather than screening processes to identify catalysts;

- $\quad$ selection for turnover rather than for affinity; and

- deployment of chemistry whose masked reactivity is inherently incompatible with cell viability.

We had in fact been close to some of these objectives in work in the early 1980s when we sought unsuccessfully to find esterase antibodies using as immunogen an aryl-trifluoromethylketone based on an irreversible inhibitor of acetylcholine esterase [14]. The concept returned, stimulated by sequential studies of Serge Halazy [15] and Kim Janda [16]. We chose to apply it to the difficult task of generating a catalytic antibody for an aryl phosphatase process, aware that Steve Withers had demonstrated the suicidal potential of $p$-difluoromethylphenyl phosphate for a tyrosine phosphatase [17]. Accordingly, we first designed and synthesized the suicide inhibitor needed for the turnover selection process (Fig. 4). In line with the work of Withers [17], this agent proved to be an irreversible inhibitor for alkaline phosphatase since phosphate hydrolysis generated an $o$-difluoromethylphenol which, on spontaneous elimination of fluoride, was converted into a suicidal quinone methide. The key feature of this essential control is that the alkaline phosphatase became irreversibly inactivated after several turnovers of the substrate [18]. Moreover, the whole process could be monitored in real time using surface plasmon resonance. 


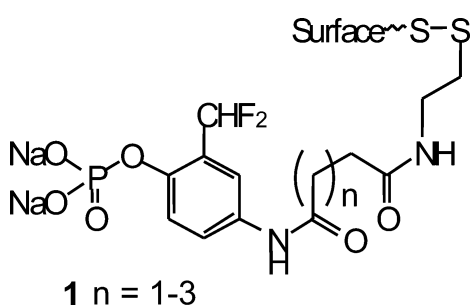

$1 \mathrm{n}=1-3$

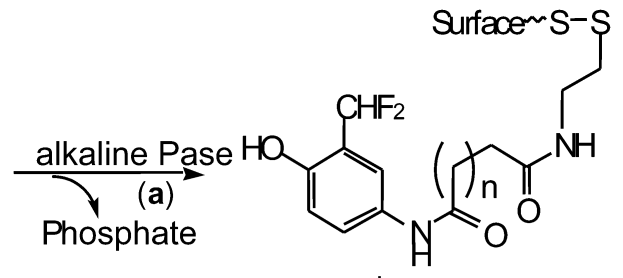

(b)<smiles>O=C(CC1CCCCC1)NCCSSCCCCCOc1ccccc1</smiles>
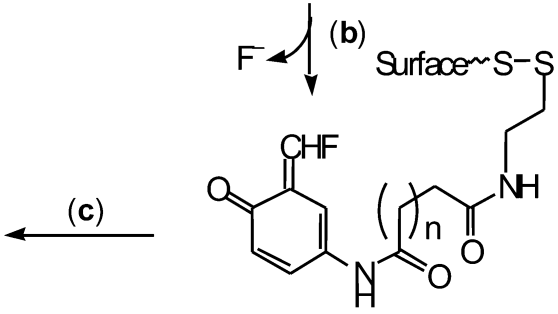

Fig. 4 Suicide substrates for alkaline phosphatase. Steps: (a) phosphate ester hydrolysis with release of phosphate; (b) loss of fluoride generates a giunone methide; (c) capture of an enzymic nucleophile in the active site binds the catalyst to the surface and inactivatives it.

We then turned to the need for a new biotechnology and engaged the wholly synthetic HuCAL fragment antibody library from Morphsys, which has 2 billion independent members from the IgG family of proteins [19]. Because this library can be accessed on the surface of a phagemid vector, the suicidal activity of the quinone methide (generated following phosphate hydrolysis) can be tolerated. It goes without saying that no mouse could withstand such an insult! The deployment of the suicide substrate (Fig. 4) as a selection reagent for the HuCAL library was immediately successful. After only two rounds of panning, several hundred candidate phages were identified capable of turnover of the suicide substrate with $K_{\mathrm{m}}$ in the range $10^{-2}$ to $10^{-6} \mathrm{M}$. A small selection of these was monitored by surface plasmon resonance in real time to identify the most efficient candidate by its immobilization to the gold surface and scFv TT1 was chosen for detailed analysis.

This single-chain protein is capable of turnover of $p$-nitrophenyl phosphate, PNPP, with an enhancement over the spontaneous rate $\left(k_{\text {cat }} / k_{\text {uncat }}\right)$ of $2.3 \times 10^{5}$. With a $K_{\mathrm{m}}$ of $46 \mu \mathrm{M}$, this corresponds to a catalytic proficiency of $5 \times 10^{9} \mathrm{M}^{-1}$, making it one of the most proficient catalytic antibodies yet described [20], and 100 times superior to the best antibody screened from a hybridoma system [21]. Because the selection process is innocent of any particular mechanism for the phosphate hydrolysis

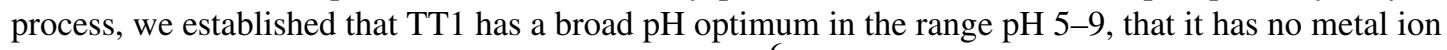
requirement, and that it is inhibited by vanadate at $10^{-6} \mathrm{M}$. It is possible to tease out some information on the mechanism by site-directed mutagenesis of the modeled binding site for aryl phosphate substrates. This identified the residues Thr-H135 and Ser-H136 as being essential for catalysis while mutation of Lys-H113, Tyr-H133, and Asp-H137 to Ala had little effect on the kinetics of cleavage.

With confidence derived from these results, we sought to improve the performance of TT1 by evolution through random mutagenesis of the whole structure with error-prone PCR. Aiming at a target of some 15 mutations per scFv chain, after 3 rounds of panning we identified 10 candidates, which proved to contain 3 copies of TT1.D1 and 2 copies of TT1.D2. These had 13 and 16 mutations respectively, distributed throughout the sequence. Both show one decade improvement in their hydrolysis of PNPP, largely through enhanced $k_{\text {cat }}$. It remains for future work to devise a strategy for directed evolution to enhance affinity of the scFvs for substrate [20]. 


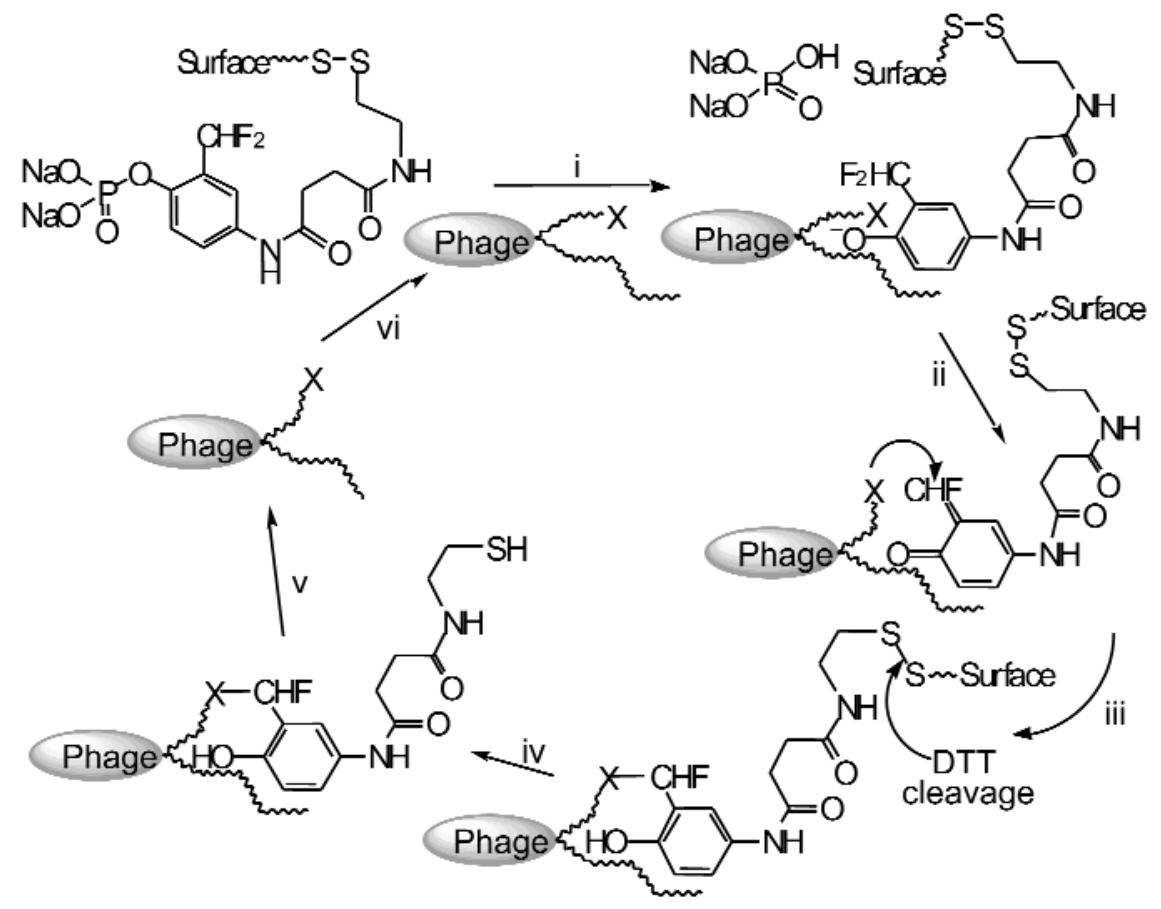

Fig. 5 Selection for turnover. (i) Catalytic antibody on phage surface binds and cleaves phosphate substrate; (ii) phenolic product loses fluoride forming a quinine methide; (iii) quinine methide bonds covalently to nucleophile (X) in/near active site of antibody; (iv) after washing away nonreactive phage, disulfide bond is cleaved by dithiothreitol to release phage from surface; (v) selected phage cloned and amplified; (vi) phage used to reenter panning cycle for refinement of catalyst selection.

\section{CONCLUSION}

This enterprise is the first example of the start-to-finish use of a man-made protein library to deliver an efficient catalyst by selection for turnover. The rates of turnover achieved match those required for conversion of a prodrug into a cytotoxic agent in vivo. Moreover, as the library has been constructed on the human immunoglobulin scaffold, it can be expected to be only weakly immunogenic in clinical application. It therefore represents the most promising approach to the ADAPT concept and the delivery of Ehrlich's "Magic Bullet" on an antibody scaffold.

\section{ACKNOWLEDGMENTS}

We thank the BBSRC for financial support (Studentship to J.H.R and Grant 50B/07047 to support A.M.); the EC for a Network Grant (ERBFMRXCT98-0193, studentships to S.C-T. and D.L.); and to MorphoSys AG for their constructive collaboration on HuCAL.

\section{REFERENCES}

1. P. Ehrlich. Lancet 172, 1634 (1907).

2. C. Milstein. Angew. Chem. 97, 819-828 (1985); G. Kohler, "Derivation and diversification of monoclonal antibodies," (Nobel Lecture), Biosci. Rep. 5, 533-549 (1985).

3. A. F. Williams, G. Galfre, C. Milstein. Cell 12, 663-673 (1977). 
4. Reviewed by I. Bernstein, "Antibody-delivered Therapy", Leukaemia Conference, 1-2 February, <http://www.webtie.org/sots/Meetings/Leukemia/02-2000/transcripts/bernstein/Transcript.htm>.

5. I. Niculescu-Duvaz. Curr. Opin. Mol. Ther. 2, 691-696 (2000); E. L. Sievers. Blood Cells, Molecules, Diseases 31, 7-10 (2003); D. L. Meyer and P. D. Senter. Annu. Rep. Med. Chem. 38, 229-237 (2003).

6. K. D. Bagshawe. Third Gordon Hamilton-Fairley Memorial Lecture, "Tumour markers-where do we go from here?" Brit. J. Cancer 48, 167 (1983). Idem, Tumor Targeting in Cancer Therapy, pp. 321-326 (2002).

7. D. C. Blakey, B. E. Valcaccia, S. East, A. F. Wright, F. T. Boyle, C. J. Springer, P. J. Burke, R. G. Melton, K. D. Bagshawe. Cell Biophys. 22, 1-8 (1993).

8. In the event, Astra-Zeneca subsequently chose to develop a carbamate prodrug for their ADEPT program as this gave a much higher enhancement of toxicity on carbamatase cleavage by CPG2. R. I. Dowell, C. J. Springer, D. H. Davies, E. M. Hadley, P. J. Burke, F. T. Boyle, FR. G. Melton, T. A. Connors, D. C. Blakey, A. B. Mauger. J. Med. Chem. 39, 1100-1105 (1996).

9. P. Wentworth, A. Datta, D. Blakey, T. Boyle, L. J. Partridge, G. M. Blackburn. Proc. Natl. Acad. Sci. USA 93, 799-803 (1996).

10. P. Wentworth, A. Datta, S. Smith, A. Marshall, L. J. Partridge, G. M. Blackburn. J. Am. Chem. Soc. 119, 2315-2316 (1997).

11. L. Pauling. Chem. Eng. News 24, 1375-1377 (1946).

12. W. P. Jencks. Catalysis in Chemistry and Enzymology, p. 288, McGraw-Hill, New York (1968).

13. J. W. Jacobs. Biotechnology 9, 258-262 (1991); J. D. Stewart and S. J. Benkovic. Nature (London) 375, 388-391 (1995).

14. G. M. Blackburn and D. R. Burton. Unpublished observations.

15. S. Halazy, V. Berges, A. Ehrhard, C. Danzin. Bioorg. Chem. 18, 330-344 (1990).

16. K. D. Janda, L.-C. Lo, L. Chih-Hung, M.-M. Sim, R. Wang, C.-H. Wong, R. A. Lerner. Science (Washington, DC) 275, 945-948 (1997).

17. Q. Wang, U. Dechert, F. Jirik, S. G. Withers. Biochem. Biophys. Res. Commun. 200, 577-583 (1994).

18. J. R. Betley, S. Cesaro-Tadic, A. Mekhalfia, J. H. Rickard, H. Denham, L. J. Partridge, A. Plückthun, G. M. Blackburn. Angew. Chem., Int. Ed. 41, 775-777 (2002).

19. A. Knappik, L. Ge, A. Honegger, P. Pack, M. Fischer, G. Wellnhofer, A. Hoess, J. Wolle, A. Pluckthun, B. Virnekas. J. Mol. Biol. 296, 57-86 (2000).

20. S. Cesaro-Tadic, D. Lagos, A. Honegger, J. H. Rickard, A. Mekhalfia, L. J. Partridge, G. M. Blackburn, A. Plückthun. Nature Biotechnol. 21, 679-685 (2003).

21. T. S. Scanlan, J. R. Prudent, P. G. Schultz. J. Am. Chem. Soc. 113, 9397-9398 (1991). 